\title{
Space charge in ionization detectors and the NA48 electromagnetic calorimeter
}

S. Palestinia ${ }^{\mathrm{a}, *, 1}$, G.D. Barr ${ }^{\mathrm{a}}$, C. Biino ${ }^{\mathrm{b}}$, P. Calafiura ${ }^{\mathrm{c}}$, A. Ceccucci ${ }^{\mathrm{a}, 1}$, C. Cerric ${ }^{\mathrm{c}}$, J.C. Chollet ${ }^{\mathrm{d}}$, M. Cirillic , J. Cogan ${ }^{\mathrm{a}, 2}$, F. Costantinic , S. Crépéd, D. Cundy ${ }^{\mathrm{a}}$, R. Fantechi ${ }^{\mathrm{c}, 2}$, L. Fayardd, G. Fischer ${ }^{\mathrm{a}}$, A. Formica, ${ }^{\mathrm{e}, 2}$, P.L. Frabetti ${ }^{\mathrm{e}, 3}$, W. Funk ${ }^{\mathrm{a}}$, A. Gianoli ${ }^{\mathrm{a}}$, S. Giudicic ${ }^{\mathrm{c}}$ A. Gonidec ${ }^{\mathrm{a}}$, B. Gorini ${ }^{\text {, }}$ G. Govi ${ }^{\mathrm{a}}$, L. Iconomidou-Fayard ${ }^{\mathrm{d}}$, V. Kekelidze ${ }^{\mathrm{f}}$, W. Kubischta ${ }^{\mathrm{a}}$, S. Luitz ${ }^{\mathrm{a}}$, I. Mannellic, M. Martinie ${ }^{\mathrm{e}}$, I. Mikulec ${ }^{\mathrm{g}}$, A. Norton ${ }^{\mathrm{a}}$, J. Ocariz ${ }^{\mathrm{d}, 4}$, D. Schinzel ${ }^{\mathrm{a}}$, M. Sozzi ${ }^{\mathrm{c}}$,

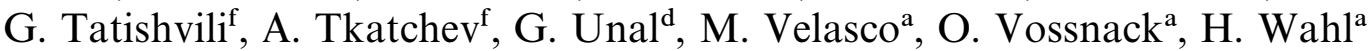

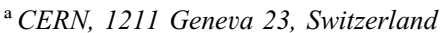

${ }^{\mathrm{b}}$ Dipartimento di Fisica dell'Università e Sezione dell'INFN di Torino, 10125 Torino, Italy

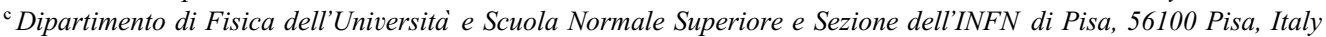

${ }^{\mathrm{d}}$ Laboratoire de l'Accélérateur Linéaire, IN2P3-CNRS, Université de Paris-Sud, 91406 Orsay, France

'Dipartimento di Fisica dell'Università e Sezione dell'INFN di Ferrara, 44100 Ferrara, Italy

${ }^{\mathrm{f}}$ Joint Institute for Nuclear Research, 141980 Dubna, Russian Federation

g Österreichische Akademie der Wissenschaften, Institut für Hochenergiephysik, 1050 Vienna, Austria

Received 24 June 1998

\begin{abstract}
The subject of space charge due to positive ions slowly moving in parallel plate ionization chambers is considered. A model for the degradation of the detector response is developed, with particular emphasis on electromagnetic calorimeters. The topics discussed include: (a) the stationary; (b) the time dependent cases; (c) the limit of very large space charge; (d) the electric field dependence of the electron drift velocity; (e) the effect of longitudinal development of showers; (f) the behaviour of the average reductions of response; and (g) the non-uniformity of response for different positions of the shower axis inside the cell defined by the electrodes. The NA48 calorimeter is used as application and for comparison of results. (C) 1999 Elsevier Science B.V. All rights reserved.
\end{abstract}

PACS: $29.40 . \mathrm{Cs} ; 29.40 . \mathrm{Vj} ; 61.25 . \mathrm{Bi}$

Keywords: Ionization detectors; Space charge; Liquid noble gases; Ion mobility; Electromagnetic calorimeters

* Corresponding author. E-mail: sandro.palestini@cern.ch.

${ }^{1}$ Permanent address: Dipartimento di Fisica dell'Università e Sezione dell'INFN di Torino, 10125 Torino, Italy.

${ }^{2}$ Present address: CEA-Saclay-DSM-DAPNIA, 91191 Gif-sur-Yvette, France.

${ }^{3}$ Permanent address: Dipartimento di Fisica dell'Università e Sezione dell'INFN di Bologna, 40126 Bologna, Italy.

${ }^{4}$ Permanent address: Departamento de Física, Universidad de los Andes, Mérida 5101-A, Venezuela. 


\section{Introduction}

Ions do not usually play a prominent role in the operation of ionization detectors, because the induced signal is essentially due to electrons, and the total amount of space charge contained in the detector cell is typically much smaller than the charge stored on the electrodes. However, the presence of positive ions becomes relevant for detectors operated at large particle intensities, or when relatively low values of the bias voltage are desired.

During the development of prototype liquid krypton calorimeters for the NA48 experiment at CERN [1], significant variations of response were observed when the intensity of an electron beam reached values corresponding to $(1-2) \times$ $10^{4} \mathrm{GeV} \mathrm{cm}^{-2} \mathrm{~s}^{-1}$, with the voltage bias set at $3 \mathrm{kV}$ over a gap of $1 \mathrm{~cm}$. The effect, which depended on the time within the beam burst, was due to space charge from positive ions. A confirmation was provided by data taken with a second prototype, which was tested at different voltage values [2].

More recently, the NA48 calorimeter has been operated at reduced voltage in a kaon beam. Effects of space charge have been observed in the central part of the detector [3].

The purpose of this paper is to review the topic of space charge effects in parallel plate detectors, to describe less intuitive aspects (nonlinearity, critically large density values), and to identify scaling variables useful to predict the behaviour in wide ranges of voltage and intensity. The formalism will be applied to the cases of detectors filled with liquid argon and, in particular, with liquid krypton, where a comparison with data collected by the NA48 collaboration will be made.

\section{Field equations}

We need first to list some considerations and assumptions:

- the drift velocity of electrons is much larger than the drift velocity of ions; as an example, electrons in liquid krypton drift at $(2-3.5) \times 10^{5} \mathrm{~cm} \mathrm{~s}^{-1}$ for electric fields above $500 \mathrm{~V} \mathrm{~cm}^{-1}$, while the drift velocity for ions is determined by the field inde- pendent mobility coefficient $(\mu)$, and is of the order of $1 \mathrm{~cm} \mathrm{~s}^{-1}$ at a few $\mathrm{kV} \mathrm{cm}{ }^{-1}$;

- we shall ignore the electron contribution to space charge, since the charge density stored in the cell gap is approximately inversely proportional to the drift velocity, as seen in detail below;

- the read-out cell is described as an infinite parallel plate capacitor;

- for the moment, the injected charge density rate $J$ is considered uniform over the cell.

Under the assumptions above, the electric field may depend on time $t$ and on the coordinate $x$ normal to the electrodes, but not on $y, z$ parallel to the electrodes. The field $E$ and the space charge $\rho$ (due to the positive ions) are determined by the equations:

$\frac{\partial E}{\partial x}=\frac{\rho}{\varepsilon}$,

$\frac{\partial \rho}{\partial t}+\frac{\partial\left(\rho v_{x}\right)}{\rho x}=J$

where $\varepsilon$ is the dielectric constant of the material filling the cell, the second equation is the charge continuity equation, $v_{x}$ is the ion drift velocity, $x=0(x=X)$ defines the anode (cathode). Using the ion mobility coefficient:

$v_{x}=\mu E$,

we obtain

$\frac{\partial \rho}{\partial t}+\mu E \frac{\partial \rho}{\partial x}+\frac{\mu}{\varepsilon} \rho^{2}=J$,

where the time evolution of the space charge is related to the ions drift (represented by the second term on the left-hand side) and to the rate of charge injection; the term quadratic in $\rho$ is due to the distortion induced by drift across regions with varying electric field, and is relevant only for relatively large $J$ and $\rho$, as discussed below. A parameter which has not appeared yet is the constraint to the integral of $E$ across the cell gap, i.e. the voltage $V$.

\subsection{The stationary case}

If $J$ is constant, $E$ and $\rho$ evolve towards a stationary case. This evolution will be illustrated in 
Sections 2.2 and 2.3. The stationary solution can be found easily from Eq. (2), in the form:

$\rho v_{x}=J x+A$,

where for the integration constant we have $A=0$ since in the limit $x=0$ (anode) there can be no accumulation of space charge (the ions drift away towards the cathode).

Using Eqs. (1) and (3) we find

$\varepsilon \mu E \frac{\mathrm{d} E}{\mathrm{~d} x}=J x$

which is directly solved as

$$
\begin{aligned}
& E=\sqrt{C^{2}+\frac{J}{\varepsilon \mu} x^{2},} \\
& \rho=\frac{J x}{\sqrt{C^{2}+J x^{2} /(\varepsilon \mu)}} .
\end{aligned}
$$

The integration constant $C$ is equal to the electric field at the anode, and is determined by the boundary condition placed by the voltage $V$.

It is convenient to use dimensionless variables:

$\tilde{C}=C \frac{X}{V}=C E_{0}$,

$\alpha=\frac{X^{2}}{V} \sqrt{\frac{J}{\varepsilon \mu}}$,

then we have

$E=\frac{V}{X} \sqrt{\tilde{C}^{2}+\alpha^{2} \frac{x^{2}}{X^{2}}}$.

Fig. 1 provides the values of $\tilde{C}$ (the normalized $E$ field at the anode) as a function of $\alpha$, together with the values of the normalized electric field at the cathode.

We see that the solution of the problem is completely described by the parameter $\alpha$ (we shall refer to this as scaling of the electric field). The distortion to the electric field $\delta E / E$ due to build up of space charge can be controlled, with increasing degrees of sensitivity, by changing the values of $J$, $V$, and $X$.

Our treatment fails at very large intensities: Eq. (7) requires $C^{2} \geq 0$, or $\alpha \leq 2$. The situation above this limit will be discussed in Section 2.3.

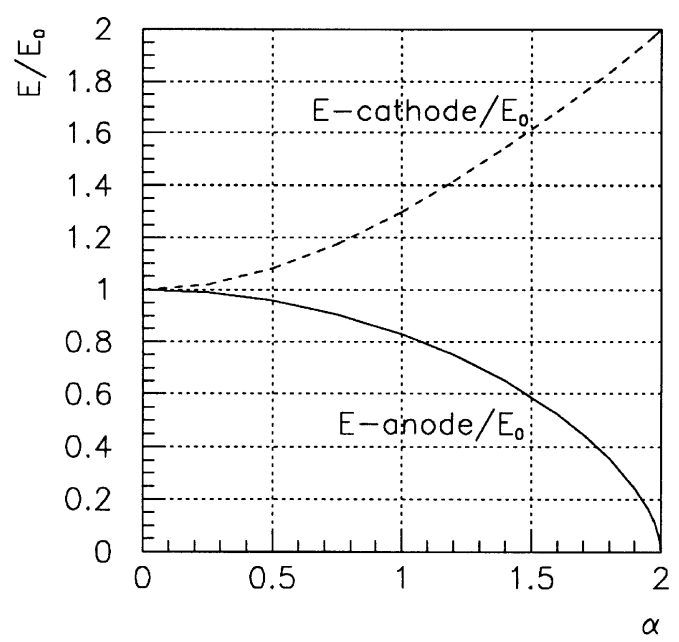

Fig. 1. Normalized electric field values at anode and cathode vs. $\alpha$.

\subsection{Time dependence}

Eq. (4) has been numerically solved. Figs. 2 and 3 show the behaviour of $\rho$ and $E$ as functions of $x$, at various values of $t$. The horizontal axis extends from the anode to the cathode. The two figures were obtained with the parameter $\alpha$ set at 1.15 and 1.99. These values are representative (for example) of the following values for the physical quantities: $\mu=0.45 \times 10^{-7} \mathrm{~m}^{2} V^{-1} \mathrm{~s}^{-1}, \varepsilon=1.5 \times 10^{-11} \mathrm{~F} \mathrm{~m}^{-1}$ (corresponding to liquid krypton), $V=1500 \mathrm{~V}$, $X=1.0 \mathrm{~cm}$; for $t<0$ we take $J=0$, while for $t>0$, $J$ is set equal to $2.0 \times 10^{-4}$ and $6.0 \times 10^{-4} \mathrm{C} \mathrm{m}^{-3} \mathrm{~s}^{-1}$, respectively, for the two cases being considered. The different lines for $\rho$ and $E$ correspond to values of $t$ for steps of $0.2 \mathrm{~s}$. The computation shows that a stationary solution is reached for time larger than about $1.5 \mathrm{~s}$.

The case of $\alpha \simeq 1.0$ is an example of low intensity conditions, where the distortion of the electric field does not exceed the $10 \%$ level, and the stationary solution shows an ion density approximately proportional to the distance from the anode. The other case $(\alpha \simeq 2.0)$ is an example of high intensity conditions, where the distortion to $E$ is larger and the space charge density flattens near the cathode, approaching a value controlled by the quadratic term in Eq. (4)

The ion density computed in the cases above is smaller than $5 \times 10^{-4} \mathrm{C} \mathrm{m}^{-3}$. In these conditions, 

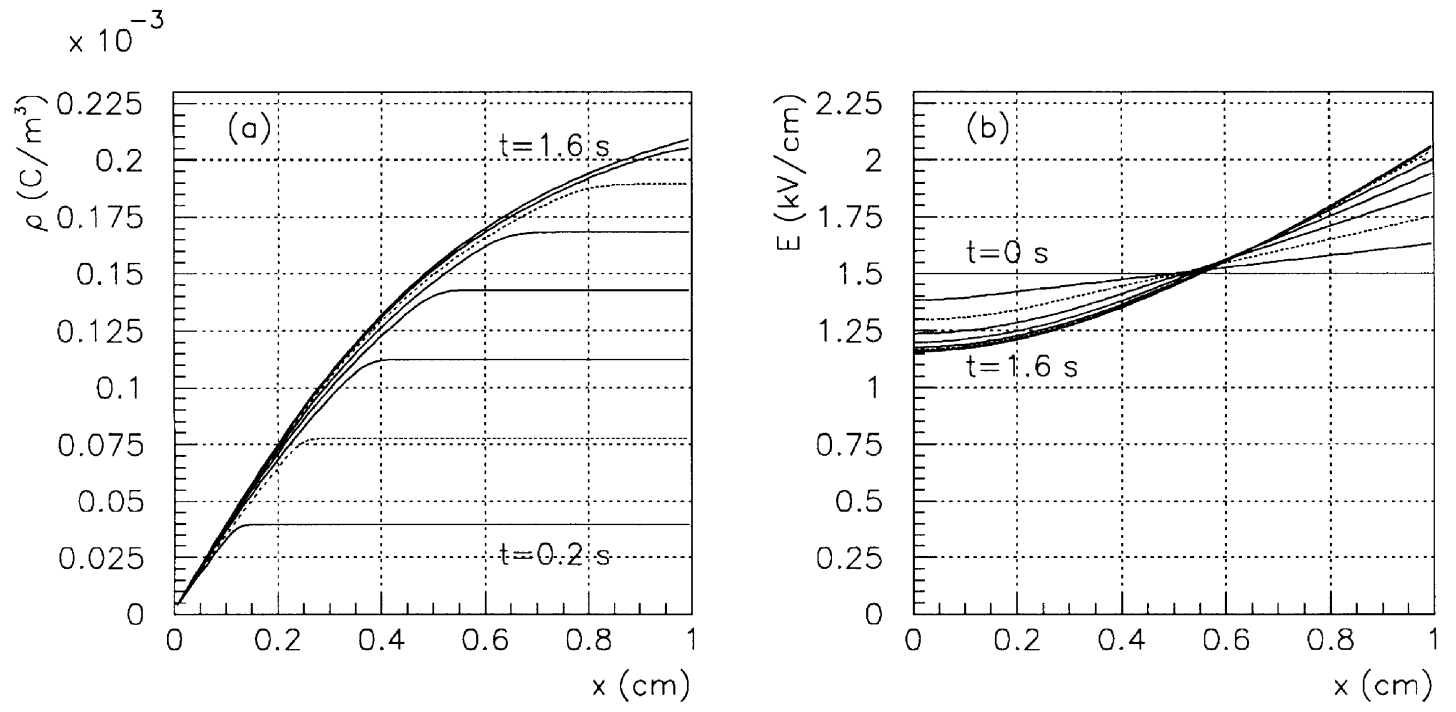

Fig. 2. Space charge density and (b) electric field vs. $x$, for different time values during the charge injection interval, for $\alpha=1.15$.
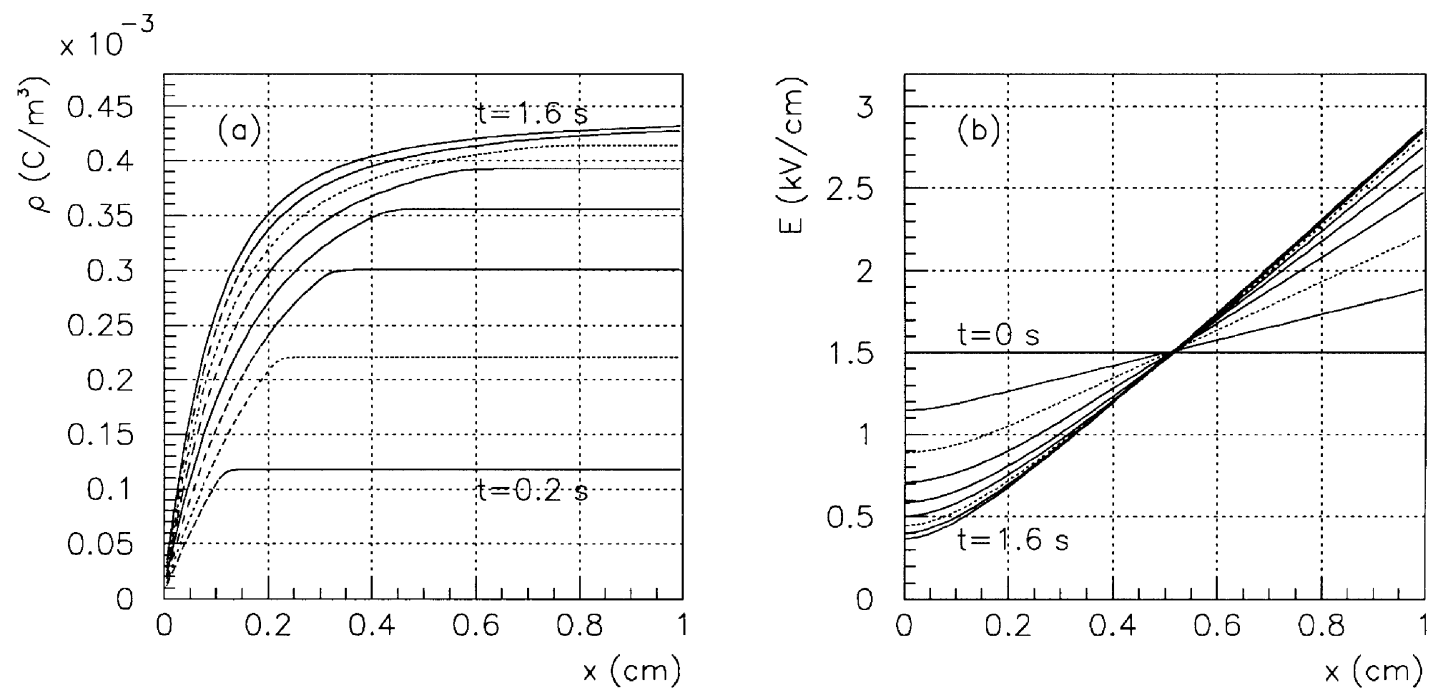

Fig. 3. Same as Fig. 2, but with $\alpha=1.99$

the electron lifetime for electron-ion recombination over long drift distances is long [4] (about $0.003 \mathrm{~s}$ in liquid krypton) compared to typical cathode to anode drift times for electrons. Therefore it is correct to ignore long range recombination in the description of space charge, as implicitly done above in Eq. (2). Recombination over short distances (free ion yield) is discussed in Section 3.1.

\subsection{Very high intensity case}

As noticed above, our formalism cannot describe cases where $\alpha>2$. The limiting (stationary) case corresponds to the constant $C$ equal to zero, with the electric field growing linearly:

$E=2 \frac{V}{X^{2}} x$ 

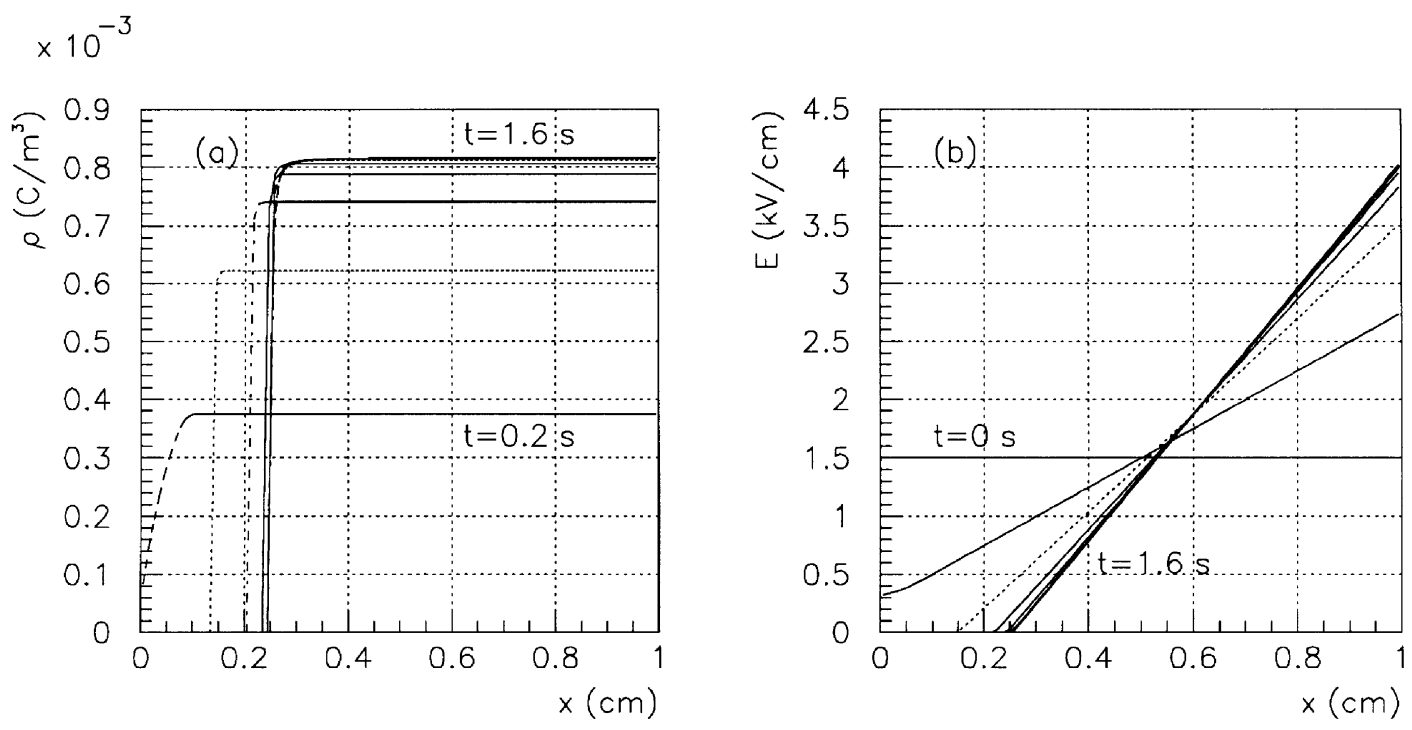

Fig. 4. Same as Figs. 2 and 3 but with $\alpha=3.6$. The contraction parameter is $\xi=0.74$.

In this condition, the ions completely shield the electric field at the position of the anode $(x=0)$. If the charge injection $J$ is further increased, the shielded region acquires a finite width. However, in regions with vanishing electric field we cannot neglect the contribution to $J$ due to electrons, which for $E=0$ do not separate from ions and cause $J$ to vanish.

Fig. 4 shows the result of the numerical integration of Eq. (4) for $\alpha=3.6$, corresponding to $J=20 \times 10^{-4} \mathrm{C} \mathrm{m}^{-3} \mathrm{~s}^{-1}$, with the other parameters set at the values given above. The stationary solution is

$$
\begin{array}{rlrl}
\rho & =0 & \text { for } x<(1-\xi) X, \\
\rho & =\sqrt{\frac{J_{\varepsilon}}{\mu}}=\frac{2 \varepsilon}{\xi^{2}} \frac{V}{X^{2}} & \text { for } x>(1-\xi) X, \\
E & =0 & \text { for } x<(1-\xi) X, \\
E & =[x-(1-\xi) X] \sqrt{\frac{J}{\varepsilon \mu}} & \\
& =[x-(1-\xi) X] \frac{2}{\xi^{2}} \frac{V}{X^{2}} \text { for } x>(1-\xi) X,
\end{array}
$$

where we have used the scale parameter:

$$
\xi \equiv \sqrt{\frac{2}{\alpha}}=\sqrt[4]{\frac{\varepsilon \mu}{J}} \frac{\sqrt{2 V}}{X} \quad(0<\xi<1) .
$$

The parameter $\xi$ describes the contraction of the active volume of the cell. For $\alpha$ larger than the critical value 2 , we find that the electric field has been removed from a part of the cell adjacent to the anode. The detector becomes a sampling device, with large variation of the electron drift velocity over the active volume.

\section{Response of an ionization chamber}

In the following sections we shall discuss how the distortion to $E$ due to space charge affects the signal extracted from the electrodes. We shall limit our analysis to the stationary case.

\subsection{Electron drift velocity}

We consider the case of initial current read-out, in which the signal from the drifting electrons is differentiated to provide a pulse of amplitude proportional to the charge $q$ of the electron cluster:

$i_{\mathrm{e}}=q \frac{v_{\mathrm{e}}}{X}$.

Space charge affects the response in the dependence of the electron drift velocity $v_{\mathrm{e}}$ on the electric field $E$. 


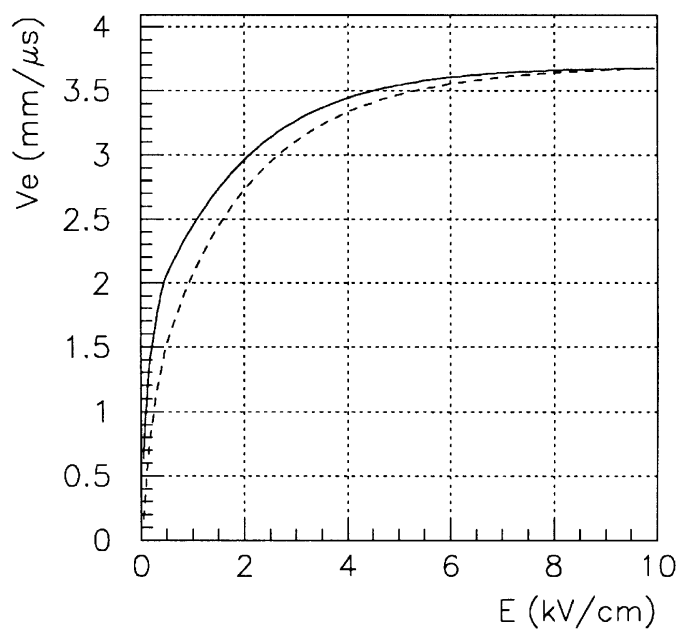

Fig. 5. Electron drift velocity vs. electric field, together with the effective value obtained by rescaling the free ion yield coefficient (dashed line)

Data on electron drift velocity in liquid krypton at $120 \mathrm{~K}$ are available [5,2]. Due to some inconsistencies we have chosen to use a parametrization based on a recent measurement made within the NA48 collaboration [6]. The effects of recombination (free ion yield), and the behaviour for very low $E$ values are included using the parametrization chosen for the NA48 simulation program [7].
Fig. 5 shows the $v_{\mathrm{e}}(E)$ dependence used for liquid krypton in this analysis, together with its value rescaled by charge recombination, which acts as an effective velocity in case of initial current read-out.

The case of liquid argon is discussed briefly in Section 3.4.

\subsection{Variations of response}

The dependence of the electron drift velocity $v_{\mathrm{e}}$ on the electric field $E$ is the key factor in determining how the distortion to the field affects the response of a detector measuring the initial current induced by drifting electrons. In the limit of linear approximation:

$v_{\mathrm{e}}(E) \simeq v_{\mathrm{e}}\left(E_{0}\right)+\frac{\mathrm{d} v_{\mathrm{e}}}{\mathrm{d} E}\left(E_{0}\right) \times\left(E-E_{0}\right)$

the response, averaged over the cell gap, is not affected by space charge, since $\langle E\rangle=E_{0}$, as imposed by the voltage applied to the electrodes, and $\left\langle v_{e}\right\rangle=v_{e}\left(E_{0}\right)$. The current induced by energy deposited near the anode is smaller than that near the cathode, because of the dependences $E(x)$ and $v_{\mathrm{e}}(E)$, but the average response does not change.

This approximation fails when $\delta E$ is such that the convexity of $v_{\mathrm{e}}$ cannot be neglected, i.e. when $\left|\mathrm{d}^{2} v_{\mathrm{e}} / \mathrm{d} E^{2}\right|$ is not much smaller than $v_{\mathrm{e}} / \delta E^{2}$. This occurs when the mean electric field $E_{0}$ is not well
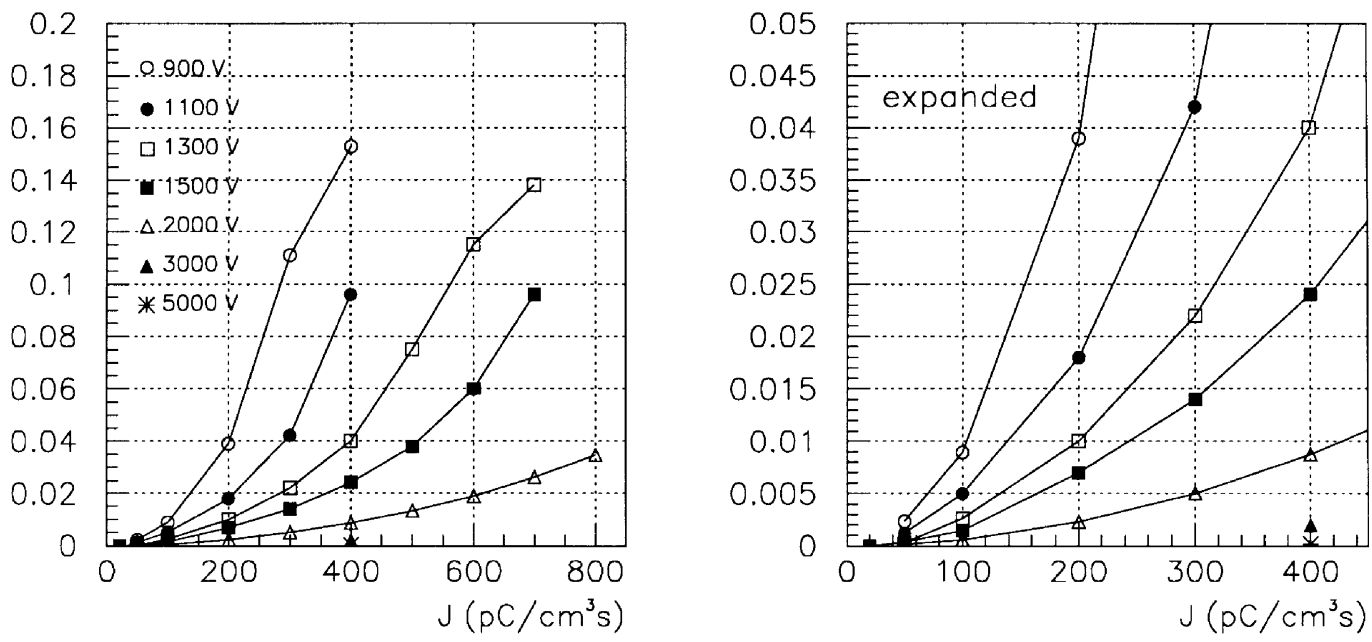

Fig. 6. Average signal reduction vs. charge injection rate for liquid krypton, with $X=1 \mathrm{~cm}$ and various voltage values. 

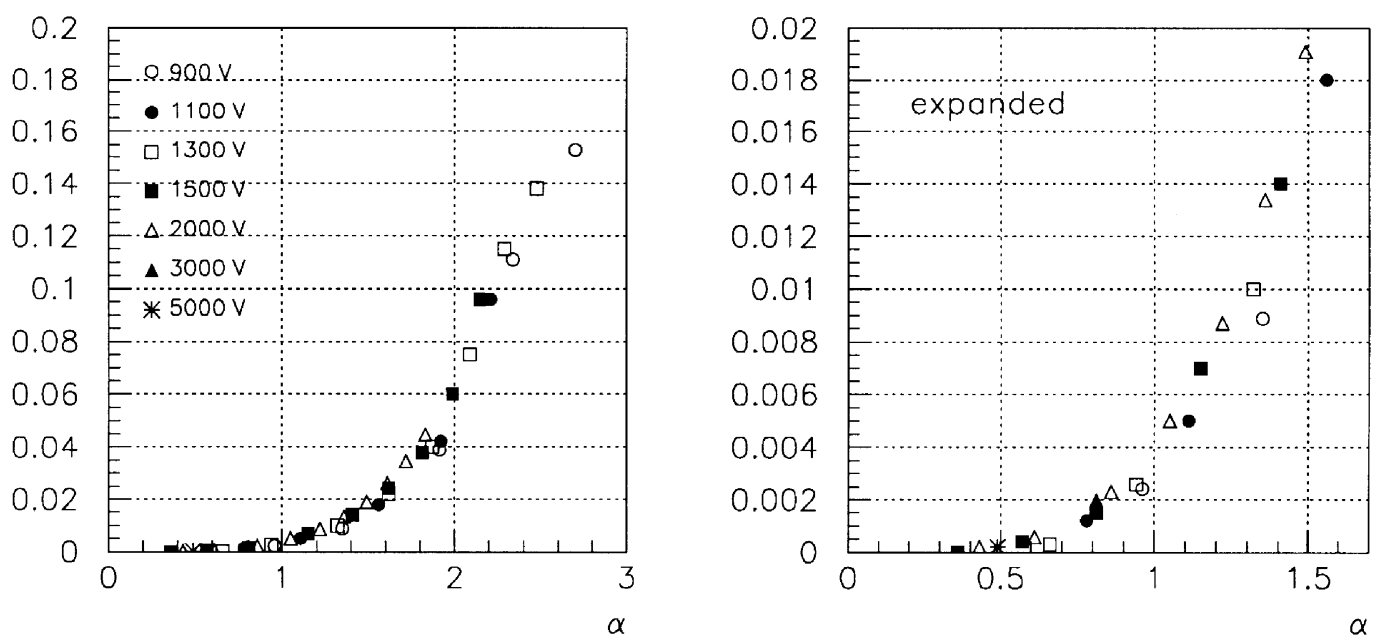

Fig. 7. Average signal reduction as a function of the parameter $\alpha$.

inside the saturation region for $v_{\mathrm{e}}$. In this case, the decrease in response near the anode is larger than the increase at the cathode, and the net result is an average reduction of signal.

\subsection{Average response}

For given values of the charge injection rate $J$ and of the parameters, $\varepsilon, \mu, V$ and $X$, the average response is obtained from the shape of $E=E(x, J)$, and the dependence $v_{\mathrm{e}}=v_{\mathrm{e}}(E)$; we consider $v_{\mathrm{e}}=v_{\mathrm{e}}(x, J)$ and average over $x$ to compute the loss of signal due to space charge. Fig. 6 shows the average signal reduction as a function of $J$, for different values of $V$, and the other parameters set at the values quoted above in Section 2.2

\subsection{Scaling behaviour of the reduction of response}

Fig. 7 shows the behaviour of the average signal reduction $(\delta)$ as a function of the variable $\alpha$. The comparison among different sets of points shows that scaling is valid at the level of $\pm 10 \%$. While the scale invariance of the electric field of Eq. (11) is a mathematical property, the scaling property of the loss of response is an approximate relation, depending on the shape of $v_{\mathrm{e}}(E)$. It is remarkable that here we find scaling to be rather accurate over a wide range of voltage values. Some points in the data sets are above the threshold for critical density $(\alpha>2)$.

An example of the dependence of $\delta$ on $v_{\mathrm{e}}$ is shown in Fig. 8, which shows a comparison of the $\alpha$ dependence of the average reduction in response computed for liquid krypton and liquid argon. The values of $v_{\mathrm{e}}(E)$ and of the charge yield in argon are from Ref. [8]. The behaviour of the reduction of response is rather similar in the two liquids.

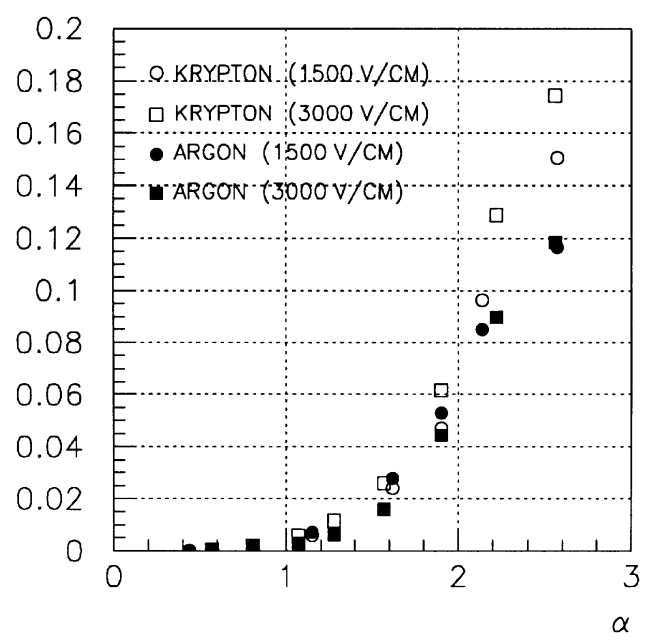

Fig. 8. Average reduction of response vs. $\alpha$ in liquid krypton and liquid argon 


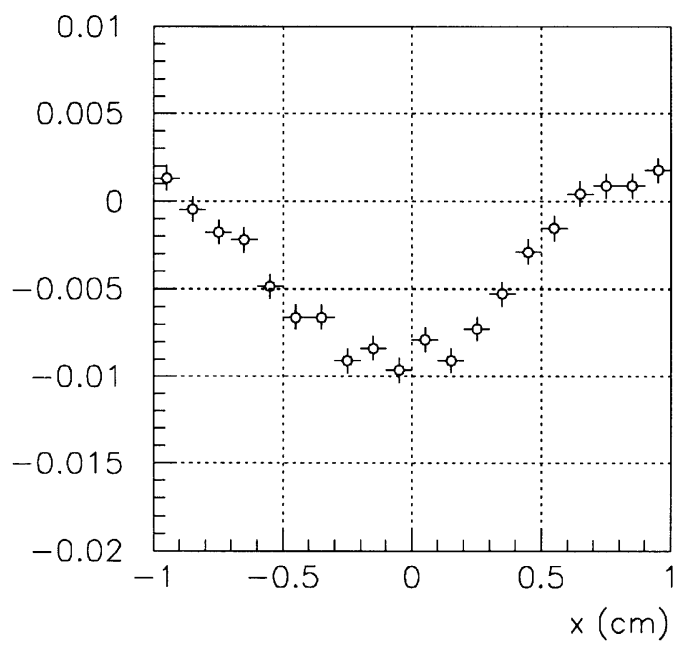

Fig. 9. Dependence of the response of the NA48 krypton calorimeter on the shower axis position within the read-out cell (space charge effect only). The value $x=0$ corresponds to the anode, and $x= \pm 1$ to the cathodes.

\section{Shower detectors}

Shower detectors will be more or less affected by space charge effects according to the main characteristics of the read-out. Calorimeters with electrodes approximately normal to the axis of the showers will be sensitive only to the average loss of response, if the detector fragmentation is high enough so that local variations of response inside the read-out gaps will be scarcely correlated with shower fluctuations. Detectors with (nearly) longitudinal electrodes will also show a dependence of the response on the transverse position of the shower axis in the cell defined by the electrode structure.

Fig. 9 shows the non-uniformity of response, due to space charge, observed with the NA48 calorimeter in the 1997 data taking period, for $20<r<25 \mathrm{~cm}$, where $r$ is the distance between the beam axis and the shower impact point [3]. The horizontal axis extends across a cell, the value $x=0$ corresponds to the anode, and $x= \pm 1$ to the cathodes. The response is defined here as the sum of the output of a cluster of $7 \times 7$ cells, each with a square cross-section with $2.0 \mathrm{~cm}$ side, and with the cells oriented along the longitudinal axis

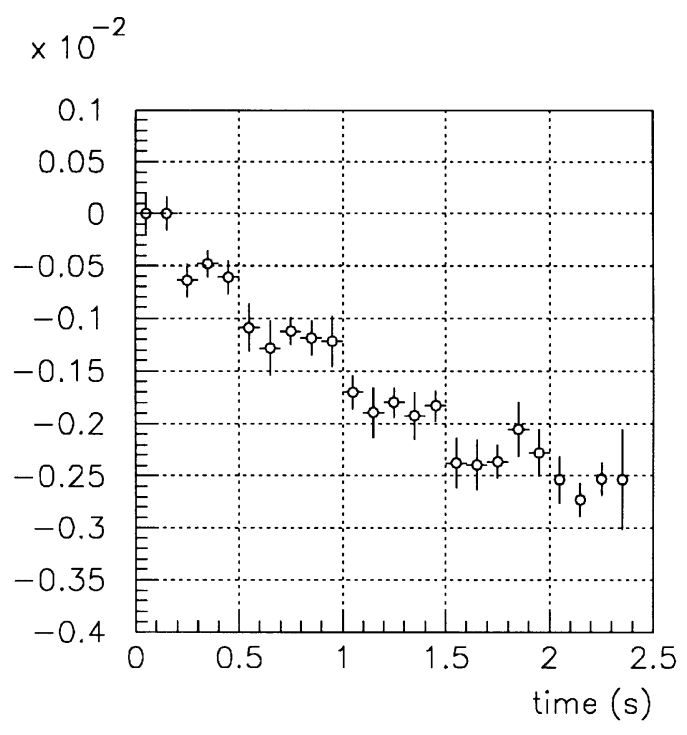

Fig. 10. Dependence of the response of the NA48 calorimeter on the shower time within the SPS extraction period.

following an accordion geometry with angles of $\pm 0.048 \mathrm{rad}$ and longitudinal segments $21 \mathrm{~cm}$ long $[2,3]$. These geometrical parameters should be compared to the radiation length $\left(X_{0}\right)$ and Molière radius of liquid krypton, both equal to $4.7 \mathrm{~cm}$. As we shall see in the following sections, space charge causes a distortion in the electric field near the electrodes equal to about $\pm 20 \%$, but because of the smearing due to the accordion angle and to the shower transverse size, the $\mathrm{x}$-dependent modulation in the response is about $\pm 0.5 \%$.

Fig. 10 shows the dependence of the response on the time of the event within the accelerator burst, averaging over the shower position, for $r<30 \mathrm{~cm}$ [3]. The average response decreases by $0.25 \%$ during the first $1.5-1.6 \mathrm{~s}$. This is the time required to reach a stationary situation with a bias voltage of $1500 \mathrm{~V}$, and, as discussed below, corresponds to an ion mobility of about $\mu=0.4 \mathrm{~cm}^{2} \mathrm{kV}^{-1} \mathrm{~s}^{-1}$. The data shown in Fig. 9 were obtained by comparing measurements made for $t<0.1 \mathrm{~s}$ and for $t>1.65 \mathrm{~s}$.

In order to apply the space charge formalism to the response of calorimeters, we need to include the longitudinal dependence of charge density. Our treatment will be mostly devoted to the average reduction in response, which will simplify part of 
the formalism, and will allow the result to be general enough to be applied to different designs of electrode structure (from transverse read-out to accordion and pure longitudinal).

In computing the average of the response $(R)$ over the position $\left(x_{0}, y_{0}\right)$ of the shower axis within the read-out cell, we effectively average over the longitudinally-integrated transverse distribution of the shower deposited charge density $(S)$. In fact

$$
\begin{aligned}
\langle R\rangle & =\left\langle R\left(x_{0}, y_{0}\right)\right\rangle_{x_{0}, y_{0}} \\
& =\frac{1}{A_{0}} \int_{A_{0}} \mathrm{~d} x_{0} \mathrm{~d} y_{0} \int_{A} \mathrm{~d} x \mathrm{~d} y S\left(x-x_{0}, y-y_{0}\right) v_{\mathrm{e}}(x, y) \\
& =\frac{1}{A_{0}} \int_{A} \mathrm{~d} x \mathrm{~d} y v_{\mathrm{e}}(x, y) \int_{A_{0}} \mathrm{~d} x_{0} \mathrm{~d} y_{0} S\left(x-x_{0}, y-y_{0}\right) \\
& =\frac{1}{A_{0}} \int_{A} \mathrm{~d} x \mathrm{~d} y v_{\mathrm{e}}(x, y) \times 1 \\
& =\left\langle v_{\mathrm{e}}\right\rangle .
\end{aligned}
$$

The equations are valid only if we can take equal and large integration areas $A=A_{0}$, which can be done without loss of generality in our definitions if the charge injection rate $J(r)$, which affects $v_{\mathrm{e}}(x, y)$, does not vary too rapidly over distances comparable to the Molière radius.

\subsection{Longitudinal development of showers}

To evaluate the effects of space charge on shower detectors, we need the longitudinal $(z)$ distribution of ions inside the detector $J(z)$, together with the longitudinal distribution of the deposited charge density $S(z)$ of the shower, which acts as a test function used to weight the decrease in response. Since the injected charge rate $J$ is due to showers, the two distributions are equal, apart from normalization factors. The average reduction in response due to space charge is given by

$\langle\delta\rangle_{z}=\int \mathrm{d} z \delta(\alpha(z)) S(z)$,

where $S(z)$ is the longitudinal shower profile,

$$
\alpha(z)=\frac{X^{2}}{V} \sqrt{\frac{J(z)}{\varepsilon \mu}},
$$

$J(z)=S(z) \times J_{L}$
The normalization factor $J_{L}$ is proportional to the energy flux into the detector. While Eq. (18) apparently implies the approximate scaling of Section 3.4, in the computation the sets of data corresponding to different values of $V$ have been used separately providing $\delta(\alpha, V)$ as different polynomial parametrizations. The integration was performed numerically, using for $S(z)$ the parametrization for e.m. showers suggested in Ref. [9].

Fig. 11 shows the result of the computations as a function of $J_{L}$, for different values of $V$ (with the other parameters set as above, representative of the NA48 calorimeter), and also as a function of the variable

$\alpha_{L}=\frac{X^{2}}{V} \sqrt{\frac{J_{L}}{\varepsilon \mu}}$

which is used in units of $\mathrm{cm}^{0.5}$. A direct comparison shows that we can reproduce rather accurately the results of Fig. 7 using for the parameter $\alpha$ an effective value:

$\alpha_{\mathrm{eff}}=\frac{\alpha_{L}}{\sqrt{13.4 X_{0}}}$.

Fig. 12 shows the decrease of response as a function of the new dimensionless variable. An approximate scaling behaviour, accurate to a few $0.1 \%$, is found for $V$ varying in the range $900-2000 \mathrm{~V}$. The length of $13.4 X_{0}$ can be taken as the depth over which the energy flux into the detector should be divided to obtain the effective charge injection density.

\subsection{A numerical example}

Using the same detector parameters considered above, valid for liquid krypton and a gap width $X=1.0 \mathrm{~cm}$, we shall apply the model to a numerical example. Consider an energy flux into the detector equal to $1 \times 10^{3} \mathrm{GeV} \mathrm{cm}^{-2} \mathrm{~s}^{-1}$. Assuming that the energy is converted into ionization in the proportion of $20 \mathrm{eV}$ per electron-ion pair, we obtain the value $J_{L}=8 \mathrm{nC} \mathrm{cm}^{-2} \mathrm{~s}^{-1}$ for the intensity parameter, corresponding to the effective charge density injection rate of $J=J_{L} / 63 \mathrm{~cm} \mathrm{=}$

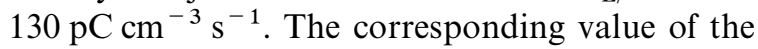
scaling parameter $\alpha$ depends on the voltage $V$, and 

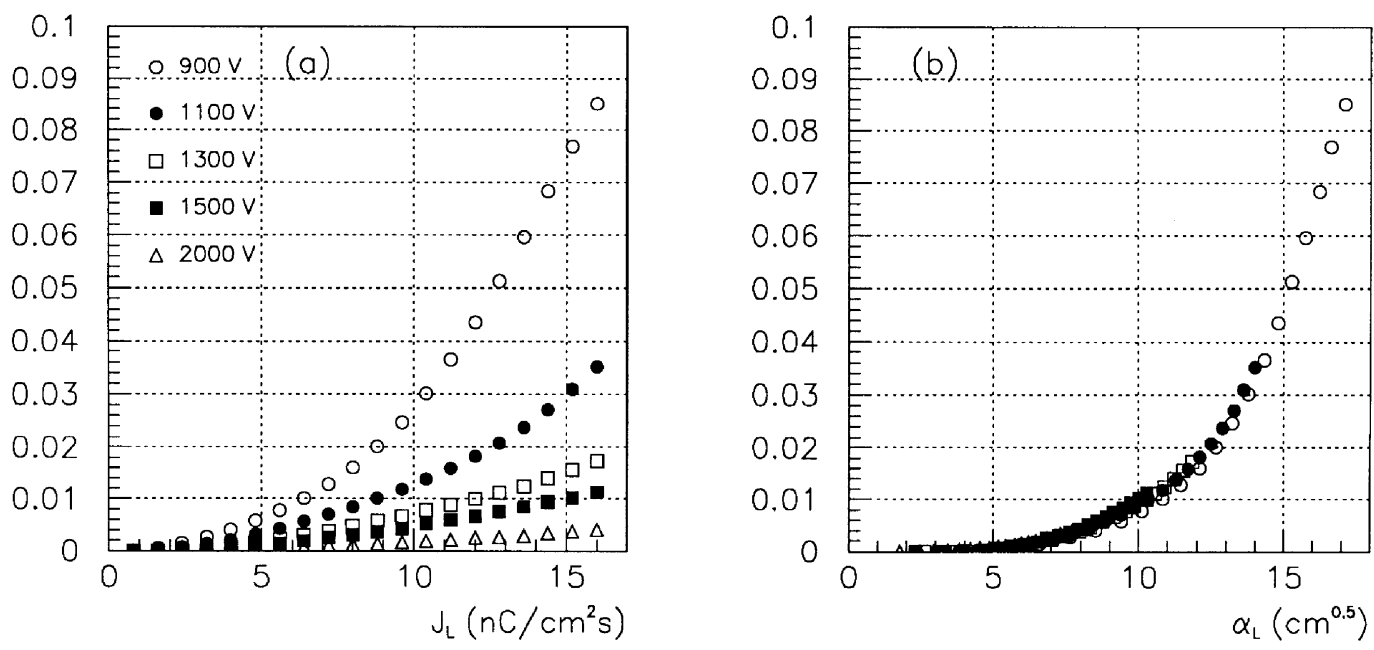

Fig. 11. Fractional decrease of response for showers, for different voltage values, vs. charge flux (a) and variable $\alpha_{\mathrm{L}}(\mathrm{b})$
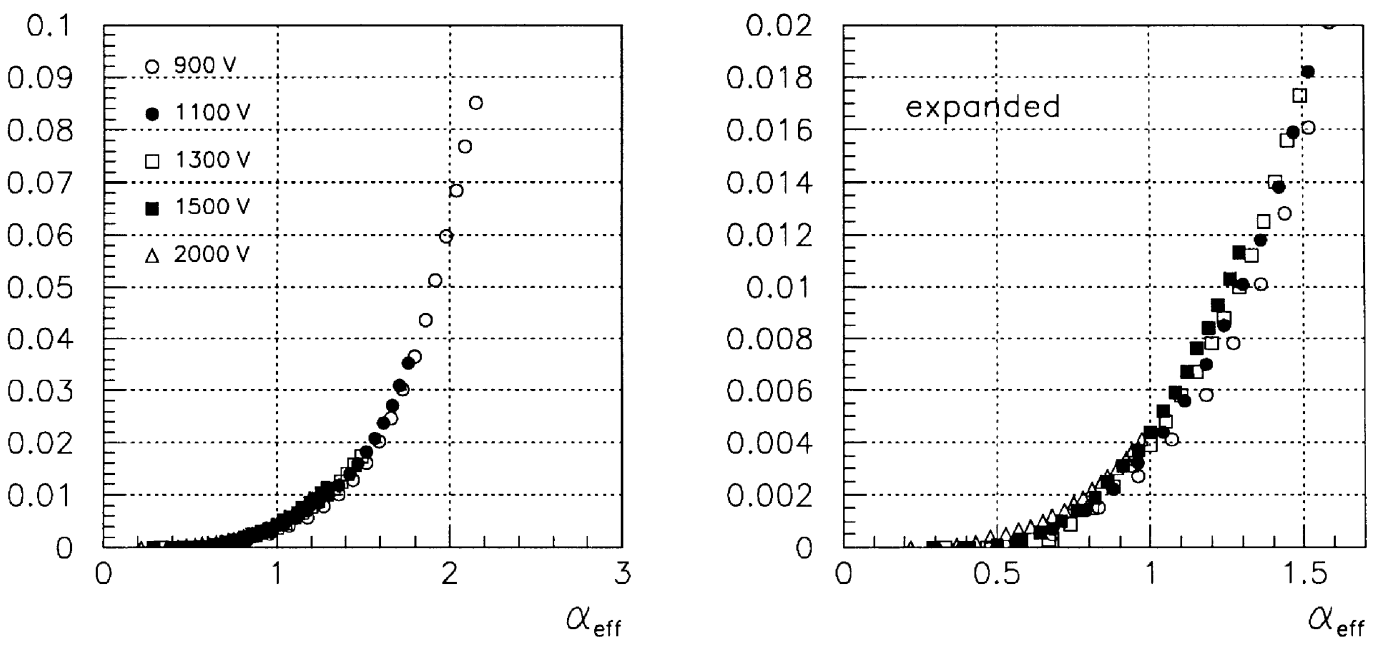

Fig. 12. Fractional decrease of response for showers vs. the variable $\alpha_{\text {eff }}$

is equal to $1.55,0.93$ and 0.70 for $V=900,1500$ and $2000 \mathrm{~V}$, respectively.

From Fig. 12, using for $\delta(\alpha)$ a parametrization which averages between the different sets of data, we see that the decrease in response due to space charge is equal to about $2.0 \%, 0.3 \%$, and $0.1 \%$ respectively for the three voltage values.

If we ignore approximate scaling and use the expression $\delta=\delta\left(J_{L}, V\right)$ Fig. 11, we find the more accurate results of $1.6,0.30$, and $0.12 \%$ for the three values of $V$. The stationary conditions would be reached after about 2.5, 1.5 and $1.1 \mathrm{~s}$ from the beginning of the extraction burst.

Repeating the computation for a charge injection value reduced by two, we would find that the values of the loss of signal are reduced by a factor in the range $3-5$. 


\section{Comparison with data}

In the following sections the predictions of the model developed above will be compared with measurements made with the NA48 liquid krypton calorimeter during the 1997 data taking period. Further details on the detector and on the data analysis can be found in Ref. [3].

\subsection{Radial dependence of the intensity}

The NA48 detector is irradiated by a neutral beam (transported in vacuum at $r<8 \mathrm{~cm}$ ) and the particles measured by the calorimeter are mostly produced in $K_{L}$ decays occurring in a region $116 \mathrm{~m}$ long, upstream of the detector. The particle rate and average energy decrease with the distance from the axis, causing the ionization injection rate $J$ to decrease rapidly with $r$. The shape of the intensity profile has been obtained using data from randomly-triggered events. Approximately $8 \%$ of the events contain at least one reconstructed shower with energy larger than about $500 \mathrm{MeV}$, in a time window of $\simeq 200 \mathrm{~ns}$. Small corrections were applied to fold into $J(r)$ the smearing due to the width of the showers, and to properly treat hadronic showers, which contribute about $20 \%$ of the total ionization, and develop over an effective length of $\simeq 100 \mathrm{~cm}$ (rather than $\simeq 63 \mathrm{~cm}$ used for electromagnetic showers).

\subsection{Intensity normalization}

The normalization of the intensity can be obtained directly from randomly-triggered events, which properly represent the particle intensity during the beam extraction from the accelerator. The sample used for this analysis amounts to 150,000 events, equivalent to $0.026 \mathrm{~s}$ of beam. The beam intensity was equal to $1.1 \times 10^{12}$ protons delivered to the primary target of the neutral beam. The mean ionization energy is equal to $20 \mathrm{eV}$, as determined from the energy to initial current ratio measured by the calorimeter read-out and its calibration system [3], and the value of the electron drift velocity at $1500 \mathrm{~V} \mathrm{~cm}^{-1}$ is $0.27 \mathrm{~cm} \mathrm{~s}^{-1}$.

Fig. 13 shows the resulting profile for $J(r)$. Uncertainties in the shape due to statistical fluctu-

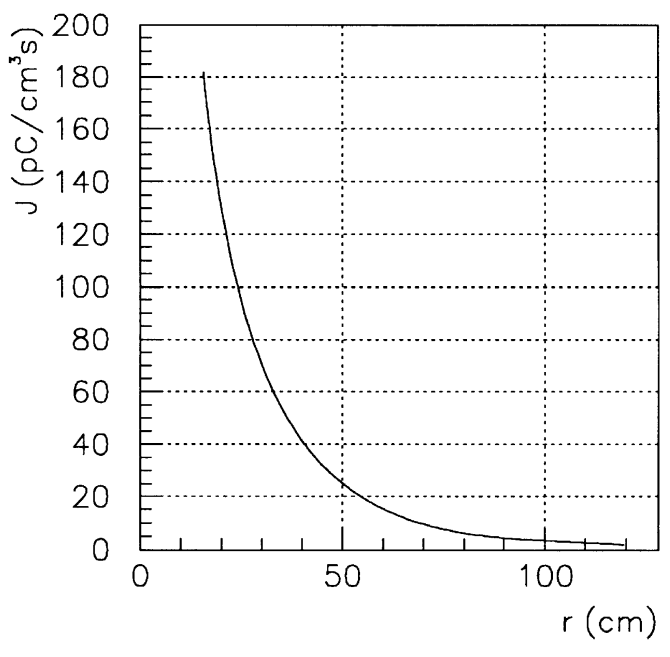

Fig. 13. Radial dependence of the effective charge injection.

ations and to energy threshold effects are at the level of $1 \%$ for $r<40 \mathrm{~cm}$ and have been neglected. The normalization error, including systematic uncertainties and burst to burst variations, is equal to $\pm 5 \%$. The value $J=130 \mathrm{pC} \mathrm{cm}^{-3} \mathrm{~s}$ at $\mathrm{r} \simeq 20 \mathrm{~cm}$ corresponds to an intensity of about $1100 \mathrm{GeV} \mathrm{cm}^{-2} \mathrm{~s}$. The intensity decreases by a factor $\simeq 100$ from $r=15$ to $r=120 \mathrm{~cm}$, and the average value over the detector face is $16 \mathrm{pC} \mathrm{cm}^{-3} \mathrm{~s}^{-1}$, or $140 \mathrm{GeV} \mathrm{cm}^{-2} \mathrm{~s}^{-1}$. This value can be compared with the measured uniform injection rate of $\simeq 1.4 \mathrm{pC} \mathrm{cm}^{-3} \mathrm{~s}^{-1}$ due to krypton radioactivity.

\subsection{Ion mobility}

Fig. 10 shows that the stationary condition, at $1500 \mathrm{~V}$, was reached $1.5-1.6 \mathrm{~s}$ after the beginning of beam extraction. This is a slightly slower approach to stationary conditions than the example shown in Fig. 2, where $\mu=0.45 \mathrm{~cm}^{2} \mathrm{kV}^{-1} \mathrm{~s}^{-1}$. The setting time is approximately equal to the time needed by the ions to drift from the anode to the cathode. In the following, we shall use $\mu=(0.41$ $\pm 0.02) \mathrm{cm}^{2} \mathrm{kV}^{-1} \mathrm{~s}^{-1}$. This value is consistent with measurements performed at higher temperatures $[10] \quad(\mu$ growing from 0.7 to $1.1 \mathrm{~cm}^{2} \mathrm{kV}^{-1} \mathrm{~s}^{-1}$ for $T$ in the range $141-184 \mathrm{~K}$ ). 


\subsection{Radial dependence of the reduction of response}

From the intensity profile of Fig. 13, the mobility value discussed above, and the expected decrease of response shown in Fig. 11, we can compute the radial dependence of the space charge effect.

Fig. 14 shows the behaviour of $\alpha_{\text {eff }}(r)$. The corresponding average reduction of response is shown in Fig. 15, where the two lines define the range determined by the uncertainties in the intensity normalization and in the value of $\mu$. As in the previous sections, we refer here to the situation after the ion density has reached a stationary condition $(t>1.5-1.6 \mathrm{~s})$, and an average over short distances is performed to remove the dependence of the response on the position of the shower within the read-out cell.

The data points in Fig. 15 are measurements performed with the NA48 calorimeter. Electrons and positrons from semileptonic kaon decays have been used to measure the reduction in the calorimeter energy measurement between events occurring for $t<0.1$ and $t>1.65 \mathrm{~s}$. About 1.3 million showers $(0<t<2.4 \mathrm{~s})$ have been used for this analysis.

It should be stressed that the curve is not a fit to the data, and its shape and normalization have been obtained from basic principles, and indepen-

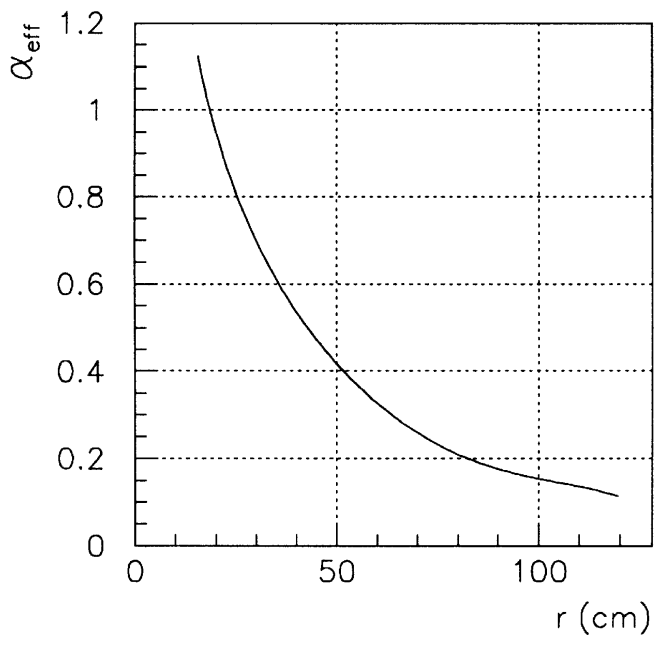

Fig. 14. Radial dependence of the parameter $\alpha_{\text {eff }}$.

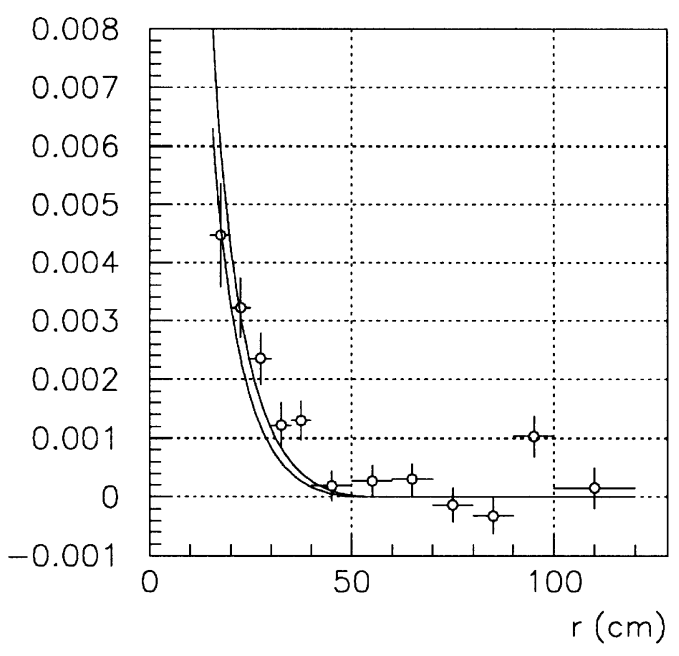

Fig. 15. Measured average reduction of response (data points) compared to the prediction.

dent measurements of intensity distribution $J(r)$ and ion mobility $\mu$. The agreement between data and model is therefore relevant and establishes confidence in the various assumptions made throughout this work.

\subsection{Fluctuations in space charge density}

Fluctuations in the ion density $\rho$ occur because of (a) variations in the beam intensity during the beam extraction burst, and (b) local statistical fluctuations in $J(r, t)$. In order to significantly change the amount of ion charge stored in the detector over a time of $\simeq 1 \mathrm{~s}$, the fluctuations (a) matter only for low frequency components of the burst time structure. Local fluctuations (b) can be estimated as follows.

If the particle spectrum at the radial position $r$ is defined by $n(E, r)$, the charge injection rate at $r_{0}$ is proportional to:

$J\left(r_{0}\right) \propto \frac{1}{A_{c}} \int_{A_{c}} \mathrm{~d} A \int \mathrm{d} E \operatorname{En}(E, r)$,

where the area $A_{\mathrm{c}}$ corresponds to the region, projected on the detector face, where the energy deposition is correlated because of the transverse size of the showers. $A_{\mathrm{c}}$ can be defined as the circular region which, for uniform $n(E, r)=n(E)$, contributes $50 \%$ 

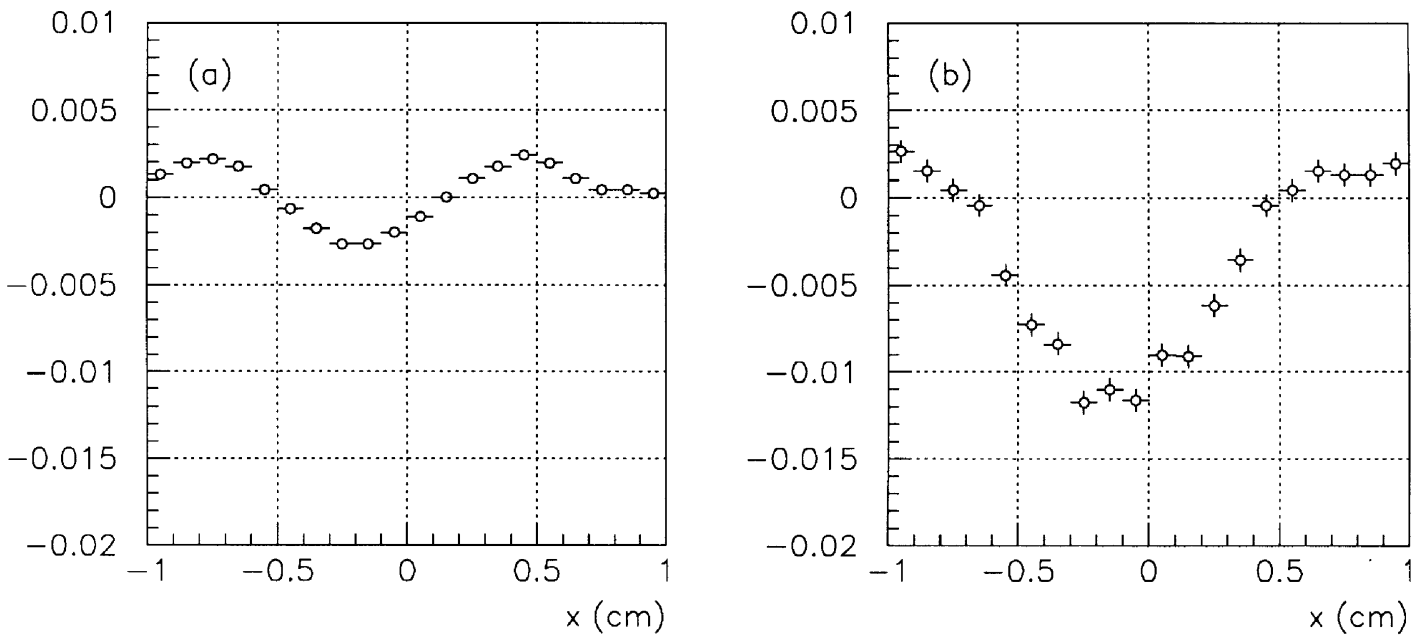

Fig. 16. Non-uniformity of detector response at low intensity (a) and at high intensity (b).

of the total ionization at its center. In liquid krypton, its area is equal to about $4.0 \mathrm{~cm}^{2}$.

The fluctuation in $J(r)$ is given by

$\sigma_{J}^{2} \propto \frac{1}{A_{c}} \int_{A_{c}} \mathrm{~d} A \int \mathrm{d} E E^{2} n(E, r)$

and the fractional deviation is related to the average values of $E^{2}$ and $E$. In the region $16<r<20 \mathrm{~cm}$ the two mean values are equal to $(22 \mathrm{GeV})^{2}$ and $14 \mathrm{GeV}$ respectively, and the number of showers collected over $1.6 \mathrm{~s}$ and $4 \mathrm{~cm}^{2}$ is equal to about 600 , corresponding to a statistical fluctuation of

$\frac{\sigma_{J}}{J} \simeq \frac{22}{14} \frac{1}{\sqrt{600}}=0.06$

The corresponding variation in the average reduction of response is $\sigma_{\delta} / \delta= \pm 0.12$, and since $\delta \simeq 0.5 \%$, the fluctuation is smaller than $0.1 \%$ and therefore of little relevance. The absolute value of the fluctuation in $\delta(r)$ is smaller at larger values of $r$.

\section{6. $x$ dependence of the space charge effect}

For completeness, this section is devoted to the reduction of response due to space charge, as a function of the position of the shower axis inside the cell of the read-out structure. Clearly, the effect depends on the geometry and read-out chosen for the NA48 liquid krypton calorimeter, and we shall not develop a general model as was done above for the response averaged over the shower axis position.

Fig. 16a shows the dependence of the detector response (here the sum over a $7 \times 7$ cells cluster) on the shower axis $X$ coordinate, in a region where space charge effects are small $(r>70 \mathrm{~cm})$. At the center of the plot $(x=0)$ the c.o.g. of the energy deposition is at the position of an anode, while the edges $(x= \pm 1)$ correspond to the positions of the cathodes. We see a modulation of the response with a peak-to-peak amplitude of $0.5 \%$. The response is smaller when the shower core is closer to the electrodes, in particular the anode, where passive material and limited bandwidth in the read-out electronics reduce the ability to extract the signal [1-3]. Since the energy-weighted longitudinal $(z)$ coordinate of the narrow shower core is different from the weighted coordinate of the entire shower, the accordion geometry of the read-out causes the position of minimum response to be shifted away from the center to the position $x \simeq-2 \mathrm{~mm}$.

Fig. 16b shows the corresponding $x$ dependence for $20<r<25 \mathrm{~cm}, t>1.65 \mathrm{~s}$, in a condition where space charge effects are large. The peak-to-peak modulation (due both to electrode effects and space charge) is now $1.4 \%$. The difference between the 

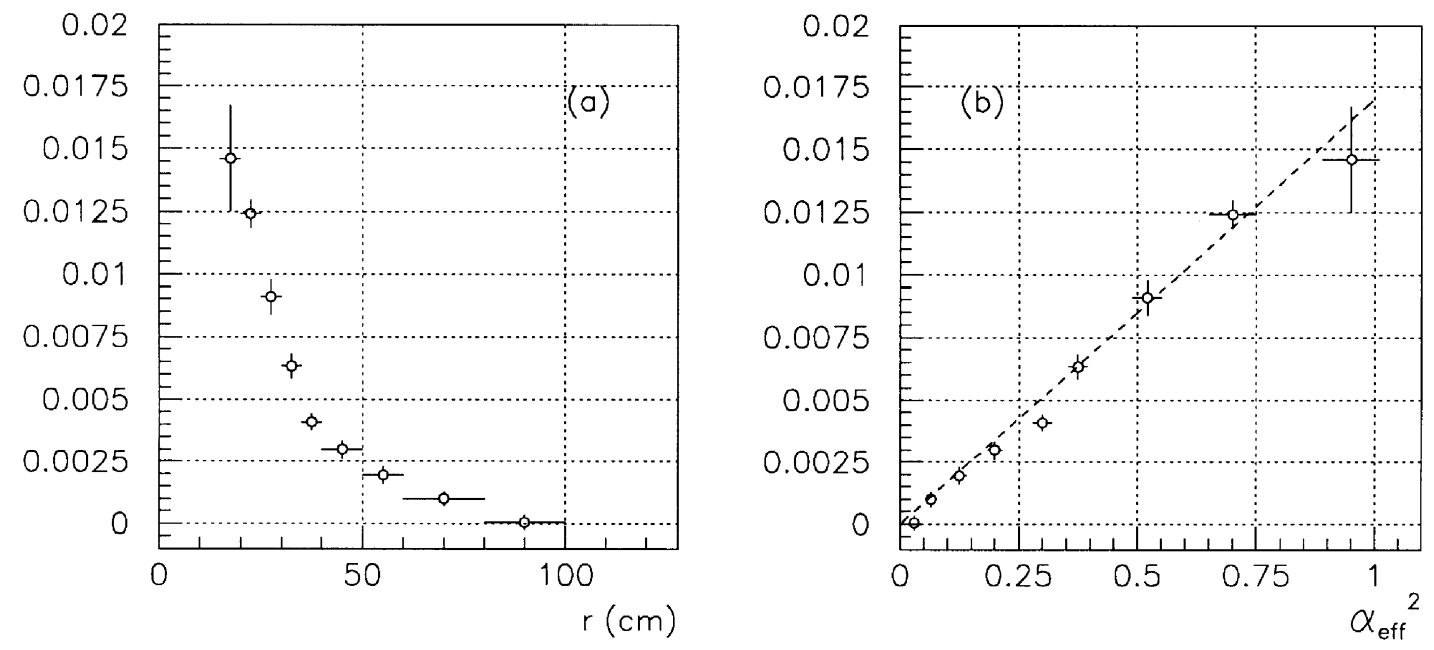

Fig. 17. Dependence of the peak-to-peak modulation of the response on the radial position (a), and on the parameter $\alpha_{\text {eff }}^{2}(b)$.

two plots, i.e. the space charge contribution only, was shown above in Fig. 9. The shape of the distortion is nearly symmetric around $x=0$.

Fig. 17a shows the behaviour of the peak-topeak modulation, for the part due to space charge only, as a function of $r$. At low values of $r$, where the intensity is high, the modulation is $\simeq 3$ times larger than the average reduction in response.

Fig. $17 \mathrm{~b}$ shows the dependence of the peak-topeak modulation on the value of $\alpha_{\text {eff }}^{2}$. A linear dependence ( $p-t-p=0.017 \quad \alpha_{\text {eff }}^{2}$ ) is found, for $\alpha_{\text {eff }}<1$, as shown by the dashed line. This dependence implies a linear relation between the modulation and the charge injection rate (namely: p-t-p $\left.=1.0 \times 10^{-4} \times J\left[\mathrm{pC} \mathrm{cm}{ }^{-3} \mathrm{~s}^{-1}\right]\right)$, and, applying approximate scaling, steeper dependences on the other detector parameters:

p-t-p $\simeq 0.017 \frac{X^{4}}{V^{2}} \frac{J}{\varepsilon \mu}$

The different regimes in which space charge affects the performance of the NA48 calorimeter can be distinguished in Figs. 15 and 17a. For $40<r<80 \mathrm{~cm}$ a small local non-uniformity of response $(0.1 \leq \mathrm{p}$-t-p $\leq 0.3 \%)$ is observed, while the average reduction of response is negligible. For $15<r<40 \mathrm{~cm}$ a larger non-uniformity is found, together with a measurable average reduction of response $(\leq 0.5 \%$ ), related to the non linearity of the electron drift velocity $v_{\mathrm{e}}(E)$. The corresponding intervals for the variable $\alpha_{\text {eff }}$ are $0.2-0.5$ and $0.5-1.1$, respectively, as shown in Fig. 14 or 17 b.

Increasing the operating voltage from 1500 to $3000 \mathrm{~V}$ brings the entire detector within the range $\alpha_{\text {eff }}<0.55$, where only minor local non-uniformities are caused by build up of space charge, and the average reduction of response is negligible.

\section{Conclusions}

A formalism for space charge effects in ionization chambers and showers detectors has been developed, identifying dimensionless parameters suited to describe a wide range in the operating conditions. Accurate and approximate scaling laws have been discussed.

The results of the computation have been compared with observations made during the operation of the NA48 calorimeter. The detector has been exposed, at reduced high voltage, to an energy flux in the range $15-1500 \mathrm{GeV} \mathrm{cm}^{-2} \mathrm{~s}^{-1}$, with the highest value localized near the beam axis. Space charge effects are visible as non-uniformity of response up to $\pm 0.7 \%$, and average reduction of response up to $0.5 \%$, in good agreement with the model predictions. 


\section{Acknowledgements}

We gratefully acknowledge the technical support from the numerous people who contributed to the design and the construction of the liquid krypton calorimeter. In particular, we thank J. Bremer for his contribution to the cryogenic system, G.F. Pagani and L. Zaccarelli for the commissioning of the front-end electronics, F. Bal, B. Hallgren, A. Lacourt, G. Laverrière, and A. Papi for their contribution to the read-out system, and G. Bocquet for her work on the off-line reconstruction code. We like to express our gratitude to all our colleagues in the NA48 collaboration for their participation in the set-up of the whole detector, in beam operation, in data collection and off-line reconstruction.

\section{References}

[1] V. Fanti et al., Nucl. Instr. and Meth. A 344 (1994) 507.

[2] G.D. Barr et al., Nucl. Instr. and Meth. A 370 (1996) 413.

[3] The NA48 Collaboration, Nucl. Instr. and Meth. A, to be submitted.

[4] S. Kubota et al., Phys. Rev. B 20 (1979) 3486.

[5] L.S. Miller, S. Howe, W.E. Spear, Phys. Rev. 166 (1968) 871; K. Yoshino, U. Sowada, W.F. Schmidt, Phys. Rev. A 14 (1976) 438.

[6] A.M. Kalinin, Yu.K. Potrebenikov, A. Gonidec, D. Schinzel, NA48 note 96-8, 1996.

[7] P. Debu, E. Mazzucato, NA48 Note 93-18, 1993. The charge yield parametrization is based on data contained in T. Stemmler, Diploma Thesis, Inst. Kernphysik Tech. Hochschule Darmstadt, 1993, and compatible with [4].

[8] V. Vuillemin et al., Nucl. Instr. and Meth. A 316 (1992) 71.

[9] E. Longo, I. Sestili, Nucl. Instr. and Meth. 128 (1975) 283; Particle Data Group, Phys. Rev D 54 (1996) 136.

[10] H.T. Davis, S.A. Rice, L. Meyer, J. Chem. Phys. 37 (1962) 947. 